\section{Everything you need to know about Quantum Optics}

possibility of generating, detecting and manipulating a variety of quantum states implies that we can control quantum fluctuations (quantum noise) as it is done in most of the topics discussed separately below.

\author{
Luigi Lugiato, University of Milan
}

Only in the eighties did the field of quantum optics begin to deserve its name fully. Previously, the quantum aspects were limited to the role of spontaneous emission in the laser and in other radiative phenomena. In the eighties fundamental concepts of quantum physics - for example, wave-particle duality, quantum state vectors, back-action in quantum measurements, uncertainty rules, mesoscopic/macroscopic superposition states (commonly called Schrödinger's cats) gradually became the playground for experimentalists in the field. Today several gedanken experiments, which we previously only found in quantum mechanics courses, are now accessible to experimentalists. At the opposite extreme, the last decade has witnessed the emergence of ideas based on new possibilities for controlling such fundamental quantum phenomena. Its unique combination of fundamental and applied aspects confers to quantum optics a special flavour making it one of the most lively and fascinating areas in physics today.

This article aims at reviewing the status of the art in this field and focussing on some recent achievements, mainly in Europe. Unavoidable are major omissions and biases introduced by my personal choices under the boundary of space limitations. The discussion is divided into different topics, but such a categorization is used as a guideline. The topics are deeply intertwined by the use of common concepts and techniques. It is also impossible to define a sharp boundary between quantum optics and laser cooling and trapping, or laser spectroscopy, which are discussed in other articles in this issue. For example, recent work of D.F. Walls and C.A. Blockley, P.Zoller pointed out the equivalence of the physics of ions in traps with the radiation-matter interaction in cavities..

A quantum concept which emerged with evidence is that of entangled states. If a quantum system $Q$ is composed of two subsystems A and B, its state is entangled when it does not factorize in the product of the states of $A$ and $B$. Even if this resembles the definition of correlation in classical physics, such quantum correlations have special and surprising aspects, related for example to the famous Einstein-Podolsky-Rosen 'paradox', in which one infers the value of the observables of A from measurements of the corresponding observables of B. Entangled photon pairs are produced, typically, in the process of parametric down-conversion, in which a single photon of frequency $\omega$ is split into a pair of photons of frequencies $\omega_{1}$ and $\omega_{2}$ with conservation of energy and momentum. The two 'twin' photons are correlated also in polarization. This technique, pioneered by Burnham, Weinberg and L. Mandel in the US, has been continued, in Europe, by A. Zeilinger, F.De Martini, J. Rarity, A. Gisin. Entanglement between photons and atoms characterizes the processes studied in cavity quantum electrodynamics, entanglement between atoms occurs for instance in the planned realizations of quantum computing with ionic traps (see below).

In the study of quantum states, one can distinguish in general three aspects.

1 Generation of nonclassical states. Nonclassical means that the state has no classical counterpart, examples are squeezed states (with Fock states as a special case), quantum correlated states, and Schrödinger's cat.

2 Detection of nonclassical states. In quantum mechanics the generation of a state can be demonstrated only by its detection. In some cases, it is enough to measure variances or correlation functions, in others it is necessary to measure probability distributions or even to reconstruct the full quantum state.

3 Manipulation of nonclassical states in view of the desired applications. What characterizes quantum physics with respect to classical is the presence of intrinsic quantum fluctuations. The

\section{Squeezing}

Let us consider any observable of a radiation beam. In classical physics the random character of the detection process implies the existence of a lower boundary to the mean square fluctuation of the observable, which is called 'shot noise limit'. In quantum physics the shot noise level corresponds to the fluctuations in the coherent states. The remarkable point is that it is possible to reduce the fluctuations of any observable arbitrarily below the corresponding shot noise level. When this happens, one says that the quantum state is 'squeezed' with respect to the observable in play. The name 'squeezed state' arises from the circumstance that, due to the Heisenberg uncertainty relations, when an observable displays fluctuations below the shot noise level, necessarily the conjugate observable has fluctuations above its shot noise level. Thus, the quantum state may be viewed as a rubber ball filled with water which, when compressed in a certain direction, necessarily expands in the orthogonal direction. This is the case, for instance, for two orthogonal quadrature components of the radiation field. In some literature the use of the word squeezing is limited to the case of quadrature components, while the definition of squeezed state given above holds for a generic observable and expresses in the best way the philosophy of squeezing, which is that of reducing as far as possible the noise in the observable of interest, without caring about the enhanced noise in the conjugate observable.

After the pioneering experiments of the groups of R.E.Slusher and of J.H.Kimble in the mid-eighties, squeezing was attained in various observables and a large number of materials. A recent achievement was obtained by the team of J.Mlynek and S. Schiller in Konstanz (in a series of experiments initiated by G.

Leuchs) with the realization of a compact all-solid state degenerate optical parametric amplifier (in which the twin photons have the same polarization and nearly the same frequency) which emits a squeezed beam with intensity quantum variance $81 \%$ below the shot noise level.

Squeezing in materials with enhanced second-order optical nonlinearity has 
been obtained by C. Fabre, by J.A. Levenson and, in the US, by Y. Yamamoto and by M. Raymer; squeezing of solitonic pulses in fibres has been achieved by $S$. Friberg in Japan, and by G. Leuchs and A. Sizmann in Germany. Intensity squeezing in semiconductor diode lasers has been studied recently by $\mathrm{E}$. Giacobino and $\mathrm{Ph}$. Grangier following the pioneering work of Yamamoto; quadrature squeezing in bulk semiconductors has been obtained by the team of A. Fox.

The applied side of the field of squeezing is often called quantum metrology, ie quantum measurements below the shot noise limit. Long baseline interferometers designed to reach the extreme sensitivity necessary for gravitational wave detection are being installed in two European projects (VIRGO and Geo6oo); similar projects are being carried in US (LIGO) and Japan. Beams with reduced quantum fluctuations can be utilised to improve the sensitivity in spectroscopic measurements; the possibility of improving polarization rotation measurements has been recently demonstrated by E. Polzik.

\section{Quantum Non-Demolition}

A central idea introduced by quantum mechanics is the notion of 'back-action': a precise measurement is not possible without the introduction of a perturbation inherent to the measurement act itself. This principle has far-reaching consequences from a practical viewpoint: the back-action induces noise in the system, that may prevent being able to retrieve the initial result in a series of repeated measurements. The concept of quantum non demolition measurements (QND) designs a measurement strategy by which the effect of back-action can be circumvented. During the eighties these ideas were realized in quantum optics, where the basic technique is to couple a signal beam, to be measured, to a second beam, called the meter beam, via an optically nonlinear medium which creates a quantum correlation between them.

In recent years, experiments have clearly demonstrated the validity ot this approach, and the recent realization of two sequential (ie repeated) back-action evading measurements by J.A. Levenson and $\mathrm{Ph}$. Grangier for pulsed waves and by S. Schiller et al for continuous waves is regarded as the ultimate proof. The present best performance of an individual QND system, achieved by the team of $\mathrm{Ph}$. Grangier, makes use of the optical nonlinearities of a cloud of trapped atoms.
These ideas have been extended to describe various techniques to manipulate and amplify information carried by an optical beam, in a way which circumvents the limitations arising from the conventional shot noise limit. These systems are robust and suited for applications, such as non-destructive read-out of information in an optical telecommunications network. Several versions of such 'quantum repeaters' have been demonstrated using either all-optical (Grangier et al) or opto-electronic (A. Karlsson and G. Biork) schemes.

\section{Quantum State Reconstruction}

While the tools for description of quantum states are well known (wave functions, density matrix, phase-space quasiprobability distribution functions such as the Wigner function), until recently it had not been known how to infer them from the results of measurements. The quantum state reconstruction methods, first introduced by H. Risken and K. Vogel, and subsequently developed by W. Schleich, A. Leonhardt and by M. D'Ariano, prescribe which observables are to be measured and how the corresponding probability distributions are to be used to permit calculation of the above quantum state descriptors. For example, in the case of a radiation field, measurements of as many quadrature components as possible are needed. A representation of the state is possible since the Fourier transform of the probability distribution of a particular quadrature is equal to a 'slice' of the two-dimensional Fourier transform of the Wigner function. This state measurement technique is called quantum tomography because it is similar to the tomography in medical diagnostics. A realization of quantum state reconstruction has been achieved by D. Wineland et al, in order to describe the oscillations of an ion in an harmonic trap, with the generation of highly nonclassical states such as Fock states or superpositions thereof, which exhibit Wigner functions with negative values. The case of modes of the light was first analyzed experimentally by M. Raymer. Later, Mlynek and Schiller reconstructed a variety of squeezed quantum states.

\section{Cavity Quantum Electrodynamics}

This field studies the radiation-matter interaction inside resonant cavities and microcavities, ie cavities with length of the order of the radiation wavelength. A major effect is that spontaneous emission is greatly modified, and can be enhanced or, conversely, inhibited in a controlled manner, as suggested by D. Kleppner in 1981.

The bulk of ideas developed in cavity quantum electrodynamics represented a development of the concepts that emerged in the sixties and seventies in the studies of lasers and of bistable absorption, but with a dramatic change in parametric conditions, which allowed such effects as lasing or bistable absorption even when in the cavity there are few atoms or, in certain cases, even less than one atom on average. This became possible thanks to the realization of cavities which are small, so that the coupling constant increases by orders of magnitude, and simultaneously of extremely high quality, ie capable of confining photons for times much longer than usual. Most importantly, in this 'strong coupling' regime radiation-matter interaction is no longer overshadowed by such irreversible processes as spontaneous emission and the escape of photons from the cavity, so that it is possible to study the fundamental reversible exchange of energy (Rabi cycles) between an atom and a cavity mode, as described by the textbook Jaynes-Cummings model. Founded by S. Haroche and $\mathrm{H}$. Walther in the microwave regime, cavity quantum electrodynamics was later extended to the optical region by H.J. Kimble and M. Feld. A crucial step was the introduction by Walther of the micromaser, in which single atoms fly through the cavity and induce the emission of a maser field with strongly nonclassical properties. The entanglement between atoms and radiation field in the micromaser is the basis of the measurement of the photon number. Very recently, this group succeeded in observing the lowest order 'trapping states', which arise when the atom-field coupling and the flight time of atoms in the cavity are chosen such that the atom undergoes one or more complete Rabi cycle under the influence of the cavity photon number, so that it leaves the cavity unchanged after the interaction. The same laboratory is working on the realization of laser emission from a single ion in a trap.

In 1996 D.J. Wineland et al reported on the observation of a Schrödinger cat generated by preparing a single trapped ion in a superposition of two distinct and spatially separated wave packets. On the other hand, a vast theoretical literature in the past studied the case of a 'photonic' cat resulting from the superposition of 

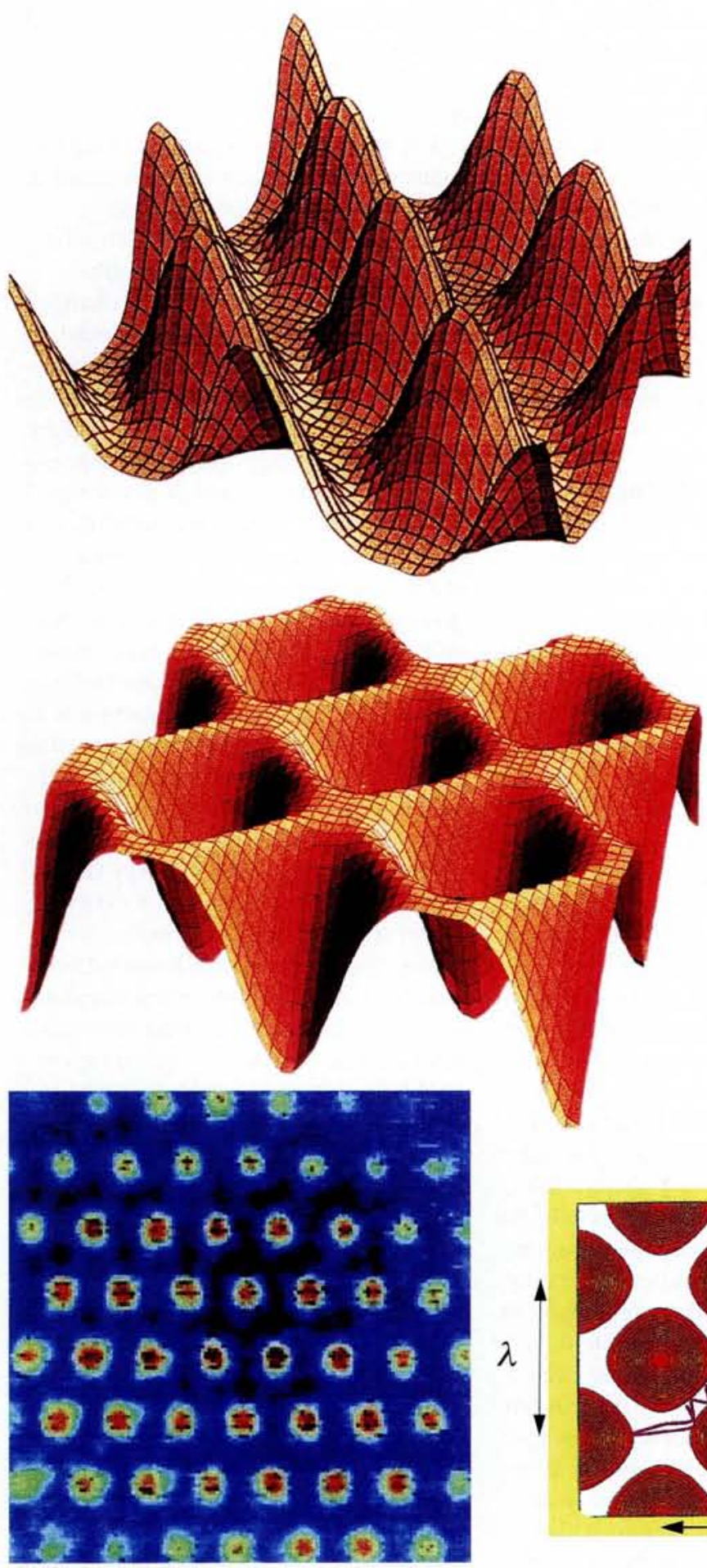

Trapped Absorption image of cesium atoms trapped in an hexagonal lattice of spatial period 29 microns. Each lattice site contains $10^{4}$ atoms at a temperature of the order of 5 microKelvin. This lattice is created by imaging a two dimensional grating with a periodic hexagonal structure

two coherent states with different phases.The group of S. Haroche and J.M. Raimond succeeded recently in realizing this cat in the field of a microvave cavity interacting with atoms that fly through the cavity itself. The difficulty in detecting
A section of the optical potential of a three-dimensional optical lattice, which consists of a periodic structure of potential wells where atoms can be trapped see below left. This lattice is created at the intersection of four laser beams tuned to the red side of an atomic transition. The localization of atoms in the potential wells has been proven by Bragg spectroscopy

An atomic lattice is lattice, which in turn is generated by interfering here is a section of the optical potential of a dot optical lattice. This lattice consists of a periodic structure of are free to move between antidots see below at the intersection of four laser beams tuned on the transition in the presence of a static magnetic field

The atomic pinball Atomic motion in an antidot lattice. Atoms in an antidot lattice bounce between antidots like balls in a pinball machine. Experimental proof of such motion has been observed with cesium atoms (All four pictures here courtesy of G. Grynberg)

cat states arises from their interaction with the environment,which makes the coherent superposition decay into a classical-looking incoherent mixture of states; this process is called 'decoherence'. For this reason, it is necessary that the photon

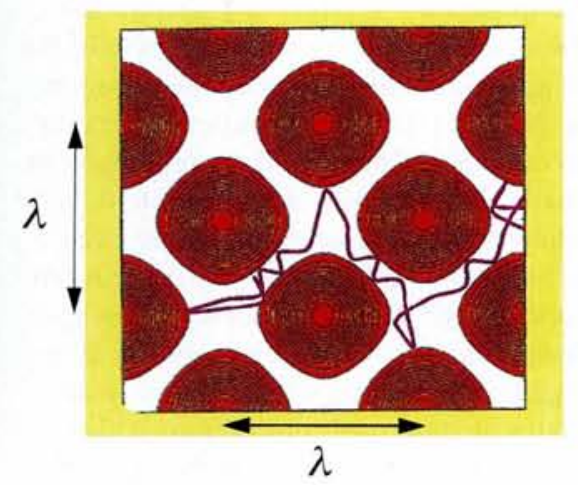

number is reasonably limited; in such mesoscopic systems one has the chance of exploring quantum physics at the boundary between the microscopic and the macroscopic world. In this experiment, the full process of decoherence was detected.

The same group recently observed Rabi oscillations in weak coherent fields, attaining direct evidence of photon number quantization in the cavity. M. Ducloy and his group studied the effects that arise from atom-photon interactions in the presence of dielectric surfaces, and the influence of the dielectric response in Cavity QED.

In the past few years, the concepts of cavity quantum electrodynamics have been extended from the domain of atomic physics to that of semiconductors. For instance, experiments by C. Weisbuch $e t$ al provided evidence of the strong coupling regime in semiconductor microcavities, in which the interaction with the radiation field is carried by excitons. This opens up the possibility of exploiting such effects in the design and operation of semiconductor optoelectronic devices. An interesting example of this approach to engineering spontaneous emission in semiconductor microcavities has been implemented recently at CNET by the group of I. Abram It is a semiconfocal parabolic cavity bounded on one side by a metallic parabolic dome and on the other by a planar dielectric mirror placed at the focal plane of the parabola.

\section{Quantum Information}

In the field of quantum information, a pioneering role has been played by the experimental tests on Bell's inequalities, carried out in the seventies by J. Clauser and in the early eighties by A. Aspect, followed by the experiments on wave-particle duality (single photon interference) of Aspect and Grangier.

The quantum world provides means with which to manipulate and transmit information, that are fundamentally different from those available in classical physics. Information processing in quantum systems can be broadly divided into three overlapping disciplines: quantum cryptography, quantum computation and quantum communication.

Quantum cryptography, or quantum key distribution, is a technique which employs a quantum optical communication channel to produce a shared and secret key (or random string of bits) between two parties. This key can subse- 
quently be used to encode messages securely. The security of quantum key distribution derives from the fact that anybody wishing to share the key must intercept the quantum signal and perform a measurement on it in order to obtain information. The legitimate users of the channel can force any eavesdropper to produce changes in the signal by adopting one of a number of appropriately designed protocols, or list of instructions. Any changes in the signal will appear as errors in the transmission and hence reveal the presence of the eavesdropper.

The theory was formulated mainly by C. Bennett, G. Brassard and A. Ekert, with following contributions eg by S.M. Barnett and S.J.D. Phoenix. On the other hand, practical quantum channels have been realized by the use of optical fibres and low intensity light pulses containing, on average, 0.1 photons per signal pulse.Ground breaking experimental and developmental work has been performed in Europe by P.D. Townsend, S.J.D.

Phoenix and K. Blow, by J. Rarity et al, and by N. Gisin et al.Current state of the art provides kilobit-per-second rates with errors at the level of $1 \%$ - which is sufficiently low to provide practical devices.

Quantum computers, which are now being envisioned, exploit the superposition principle in order to provide a new and intrinsically quantum form of computational process. A quantum computation can process all possible input data strings in one running of the algorithm, rather like a massively parallel computer. The new feature, however, is that a single register is placed in a superposition of all possible data strings and that the subsequent processing exploits quantum interference to enhance the probability that the register evolves through the computation towards the required quantum state corresponding to the required answers. It has been shown that quantum computers can address time consuming problems, like factoring large numbers and searching large databases, significantly more quickly than any system based on classical computer logic. The theory of these systems has been formulated mainly by D. Deutsch, P. Shor and A. Ekert on the 'algorithmic' side, and by P. Zoller and I. Cirac on the implementation side. A. Ekert, P. Knight, A. Steane and others have developed error-correction and stabilization techniques, as well as physical and algorithmic methods of protecting quantum states against decoherence.

Unlike quantum cryptography, quan- tum computers are a long way off, but experiments based on nuclear magnetic resonance and on strings of cooled and trapped ions have been proposed which should make it possible to realize and study simple quantum logic elements. Quantum logic gates have been realized by the groups of D.J.Wineland and of H.J. Kimble.

As for quantum communication, the focus of attention is now the recent experimental realizations of quantum teleportation obtained by the groups of F. De Martini (Rome) and of A. Zeilinger (Innsbruck). This phenomenon was predicted in 1993 by C. Bennett and collaborators. In classical physics, a transmitter A can allow a distant receiver $B$ to reconstruct an object, by simply measuring all the properties of the object and transmitting them to B. In quantum physics, on the other hand, it is not possible to know completely the state of any object. It is possible, however, to transfer an unknown quantum state from A to B. For definiteness, let us consider single photon polarization states. The key point is that $\mathrm{A}$ and $B$ must each have one of a pair of entangled photons. A performs a special kind of joint measurement of his entangled photon and of the photon whose state has to be teleported. This measurement projects the two photons in a state that does not contain any information about the initial state of the teleportee, ie all the information about the states of the two photons are erased (the possibility of a 'quantum eraser' was predicted by M.O. Scully many years go). Such a measurement can lead to four possible results and A communicates to B, via a classical channel, which of these results has been found. Depending on the result, B must perform one of four unitary transformations on the polarization of his entangled photon which, as a consequence, is carried exactly to the original state of the teleportee photon. This state remains unknown to both $\mathrm{A}$ and $\mathrm{B}$.

Even if none of the two experiments corresponds to this ideal scheme completely, they outline the core steps. The Rome experiment is, more precisely, the realization of a scheme suggested by S. Popescu; in a related experiment, again in Rome, results exceeded what could be achieved classically by 8 standard deviations. In Innsbruck, the teleportation experiment followed the demonstration of the possibility of 'quantum dense coding', ie transmission of two bits of information by a single photon. And it was

\section{TOP SECRET}

\section{Quantum Cryptography for Real World Applications}

Quantum methods of providing an unconditionally secure channel for communication are becoming increasingly practical. But will they be able to meet the demands from 'real world' applications?

The last decade has seen the birth of quantum information, the marriage of quantum physics and information technology. Of various proposals and experimental demonstrations, quantum cryptography is the closest to real world application. In secret key cryptography two parties can hide their message from outsiders provided that they share some initial secret information - the key.

In quantum cryptography, or quantum key distribution, the secret key is sent encoded on single photon pulses, in such a way that the Heisenberg uncertainty principle guarantees the detection of an eavesdropper. The classical cryptography method popular today, so called public key cryptography, bases its security on complex theoretical assumptions, essentially the difficulty of factoring large numbers, and it is not yet fully known if factoring is fundamentally difficult. If it is shown not to be, then public key cryptography will fall apart, perhaps leaving quantum cryptography to be the only practical method of key distribution.

From the initial 'proof-of-principle' demonstration in 1989 of secret key transmission over 30 centimetres by researchers at IBM (see Bennet in further reading), the field has progressed remarkably. At Los Alamos National Laboratories secret keys have been transmitted in optical fibres over distances of 48 kilometres, and up to 1 kilometre in free space. BT labs have sent cryptographic keys in a 1.3/1.5 micrometer field system, and the University of Geneva have demonstrated a very stable 'plug and play' interferometric system on 23 kilometres of installed telecom 
recently followed by the realization of 'entanglement swapping' as predicted by M. Zukowski $e t$ al and by P. Knight $e t a l$. In this case, the teleported photon is originally entangled with another photon P. Teleporting this state transfers the entanglement to B's photon, even if it never interacted, or shared a common past, with P. In these experiments, teleportation occurs over a distance of the order of a meter; however, it should be possible to increase the distance notably because $\mathrm{N}$. Gisin et al demonstrated recently entanglement of photons over distances as great as 10 kilometres. The teleportation of atoms should also become possible following the experiment of the group of S. Haroche and J.M. Raymond that demonstrated entanglement of pairs of rubidium atoms.

\section{Spatial Quantum Structures}

Radiation beams with large sections, interacting with nonlinear optical materials, generate spontaneously a wide variety of spatial structures which are studied in the field of optical pattern formation. Unlike similar structures in other fields such as, for example, hydrodynamics or nonlinear chemical reactions, optical patterns can show noteworthy quantum aspects, even at room temperature. For instance, only quantum theory is capable of predicting the emission of down-converted photons in optical parametric amplifiers and optical parametric oscillators below threshold. Together with A. Gatti and G-L. Oppo, I have shown that such a down-converted field can exhibit a notable level of spatial order; the name 'quantum images' was coined to designate these types of noisy spatial patterns. Looking at the spatial correlation functions one identifies precise signatures of the quantum nature of the fluctuations that generate the image. In this context entanglement phenomena, with their Einstein-Podolsky-Rosen aspects, can be studied from a spatial viewpoint, for macroscopic and spatially extended radiation beams instead of single photon pairs. The spatial aspects of squeezing can be investigated and exploited for the observation of faint images, as shown first by M. Kolobov and I.V. Sokolov. C. Fabre is studying the application of such phenomena to the detection of edges on the nanometric scale; B.Saleh is developing an approach to 'quantum imaging'.

In atomic physics one can find fascinating spatial patterns. In the late eighties, $\mathrm{H}$. Walther and his group realized micro- crystals formed by a small number of ions in a trap, observed via their fluorescent emission. When their kinetic energy is increased, the crystal melts and one has a chaotic motion. Linear arrangements of ions in storage rings have also been obtained in the same laboratory. The groups of Walther and of R. Blatt are presently realizing linear configurations tailor made for application to quantum computing. Atomic lattices, on the other hand, constitute a new form of matter which combines the crystalline order of a solid with the density of a high vacuum. They are created by the interference pattern of a number of intersecting laser beams; according to the number and the arrangement of the beams, such a spatially modulated optical lattice has a $1 \mathrm{D}$, or $2 \mathrm{D}$, or $3 \mathrm{D}$ structure. Each individual element of this optical lattice works as a trap for cold neutral atoms; the atomic lattice is formed by a collection of atoms in different sites of the optical lattice. In contrast to solid state physics, the atomic lattice can be controlled and modulated from the outside. Such patterns, realized in the laboratories led by G.Grynberg in Paris, Th. Haensch in Munich and W.D. Phillips in Gaithersburg, provide a novel nonlinear optical medium that has been analyzed extensively. In addition to such 'bright lattices', also 'grey lattices', in which the atoms emit only few photons, have been created by those groups. Grynberg et al discovered a pinball-like motion of atoms in grey lattices (see illustrations on page 133), and realized lattices with a quasiperiodic (instead of periodic) structure. Haensch et al have measured the spatial spread of the atomic wave packet, and observed breathing oscillations after a sudden change in the profile of the optical lattice. Walther et al studied the motion of a single ion in an optical lattice, observing significant deviations from the standard diffusion law.

By lowering the temperature, it becomes possible now to investigate the quantum aspects of atomic lattices. Haensch and collaborators realized recently a configuration in which the micro-traps of the optical lattice are highly non-dissipative and much larger than usual, so that many atoms can be loaded into each site, achieving phase space densities less han three orders of magnitude away from Bose-Einstein condensation. Both nonlinear optical patterns and atomic lattices provide an appropriate framework to study coherent quantum phenomena. 